\title{
RACSAM
}

Rev. R. Acad. Cien. Serie A. Mat.

VOL. 103 (1), 2009, pp. 167-175

Matemática Aplicada / Applied Mathematics

\section{Stabilization beyond the distributions}

\author{
J. I. Díaz and E. Sanchez Palencia
}

\begin{abstract}
We prove that for suitable evolution problems, the solution $u(t)$ corresponding to some right hand side term $f(t)$ in $V^{\prime}$ (with $V$ some Hilbert space), only satisfies the stabilization property $\left(f(t) \rightarrow f_{\infty}\right.$ in $V^{\prime}$ implies that $u(t) \rightarrow u_{\infty}$, in $V$, when $t \rightarrow+\infty$, with $u_{\infty}$ solution of the associated stationary problem) when the space $V$ is taken strictly larger than the distribution space. This type of problems arise, for instance, in the study of some quasi-stationary viscoelastic shell-like problems in the presence of friction effects.
\end{abstract}

\section{Estabilización más allá de las distribuciones}

Resumen. Probamos que en ciertos problemas de evolución, la solución correspondiente a adecuados términos del lado derecho $f(t)$ en $V^{\prime}$ (con $V$ cierto espacio de Hilbert), sólo satisface la propiedad de estabilización $\left(f(t) \rightarrow f_{\infty}\right.$ en $V^{\prime}$ implica que $u(t) \rightarrow u_{\infty}$, en $V$, cuando $t \rightarrow+\infty$, con $u_{\infty}$ solución del problema estacionario asociado) cuando el espacio $V$ es tomado estrictamente más grande que el espacio de distribuciones. Este tipo de situaciones aparecen, por ejemplo, en el estudio de ciertas formulaciones del estilo de los problemas de estructuras finas viscoelásticas con fricción.

\section{Introduction}

The fundamental role of Distribution Theory, offering the correct framework in which most of the models of the Mathematical-Physics must be formulated is well-known in our days ([11, 5, 8, 7]). Nevertheless, there is a large amount of singular problems, (arising in many different contexts as, e.g. in thin shell theory) which lead to formulations beyond the distributions.

The main goal of this article is to present some results showing that, even in this case, it is possible to show the stabilization, as $t$ goes to infinity, in a context more general than the space of distributions. In fact, this philosophy has many common points with a series of papers dealing with some singular stationary problems (see, e.g. [9] and [6]). Our main contribution is to replace the direct role played by a parameter $\epsilon$ (which in the above mentioned papers is assumed converging to zero) by the derivative with respect to time when we consider suitable evolution problems and we assume that the time $t$ converges to infinity.

In a first part of the paper (see Section 2) we consider an academic problem for which we can prove directly the stabilization of solutions beyond $\mathcal{D}^{\prime}$;

$$
\left\{\begin{array}{l}
\frac{\mathrm{d} u}{\mathrm{~d} t}(t)+A u=f(t) \quad t>0, \text { in } V^{\prime} \\
u(0)=0
\end{array}\right.
$$

Presentado por / Submitted by Amable Liñán Martínez..

Recibido / Received: 11 de octubre de 2008. Aceptado / Accepted: 14 de enero de 2009.

Palabras clave / Keywords: Stabilization, more general than distributions, like-shell problems.

Mathematics Subject Classifications: 35B40, 35K90, 35S10, 74H40.

(C) 2009 Real Academia de Ciencias, España. 
where $V$ is a Sobolev space on $\Gamma$, a one-dimensional compact manifold without boundary (for instance a circle), $A=S^{*} S$ and $S$ is a suitable smoothing operator, i.e. verifying that $S\left(H^{s}(\Gamma)\right) \subset H^{r}(\Gamma)$ for any $s, r \in \mathbb{R}^{+}$(in particular $S\left(\mathcal{D}^{\prime}(\Gamma)\right) \subset C^{\infty}(\Gamma)$ ). Here $S^{*}$ denotes the adjoint of $S$.

In a second part (see Section 3) we consider a problem related, in some sense, to shell theory. We consider a special formulation for the transient displacements of a thin shell under a viscoelastic constitutive law. Then, following the general approach by G. Duvaut, and J. L. Lions [4, Section 6, Chapter 3] and J. Sanchez-Hubert and E. Sanchez Palencia [10] we arrive to a formulation of the type

$$
\frac{\partial^{2} \mathbf{U}}{\partial t^{2}}+c\left(\frac{\partial \mathbf{U}}{\partial t}, \mathbf{v}\right)+a(\mathbf{U}, \mathbf{v})+\varepsilon^{2} b(\mathbf{U}, \mathbf{v})=\langle\mathbf{f}, \mathbf{v}\rangle
$$

for suitable test functions in the energy space $V$ (a certain Sobolev space) and bilinear forms $a, b$, and $c$. The so called quasi-static problem corresponds to

$$
c\left(\frac{\partial \mathbf{u}}{\partial t}, \mathbf{v}\right)+a(\mathbf{u}, \mathbf{v})+\varepsilon^{2} b(\mathbf{u}, \mathbf{v})=\langle\mathbf{f}, \mathbf{v}\rangle
$$

and it provides a reasonable good approximation for the asymptotic time since it was shown in [4] that (under suitable conditions: see Corollary 6.1, Chapter 3) that

$$
\left\{\begin{array}{l}
\|\mathbf{u}(t, \cdot)-\mathbf{U}(t, \cdot)\|_{V} \leq C \mathrm{e}^{-\gamma t} \quad \text { and } \\
\mathrm{e}^{\gamma t}\left(\frac{\partial \mathbf{u}}{\partial t}-\frac{\partial \mathbf{U}}{\partial t}\right) \in L^{2}(0,+\infty: V),
\end{array}\right.
$$

for some $C, \gamma>0$.

The main goal of this work is to analyze the stabilization of solutions of (1), as $t \rightarrow+\infty$, to the solutions $\mathbf{u}_{\infty}^{\epsilon}$ of the stationary problem

$$
a\left(\mathbf{u}_{\infty}^{\epsilon}, \mathbf{v}\right)+\varepsilon^{2} b\left(\mathbf{u}_{\infty}^{\epsilon}, \mathbf{v}\right)=\left\langle\mathbf{f}_{\infty}, \mathbf{v}\right\rangle .
$$

Moreover, as it can be shown (following the ideas introduced in the papers [9] and [6]) the solutions $\mathbf{u}_{\infty}^{\epsilon}$ of (2) converge, when $\epsilon \rightarrow 0$ (in a functional space which is not included in the distributions space $\mathcal{D}^{\prime}$ ), to a solution $\mathbf{u}_{\infty}$ of

$$
a\left(\mathbf{u}_{\infty}, \mathbf{v}\right)=\left\langle\mathbf{f}_{\infty}, \mathbf{v}\right\rangle .
$$

So that the double limit, as $t \rightarrow+\infty$ and $\epsilon \rightarrow 0$ leads the solutions of (1) to the solutions of (3) in a functional space beyond $\mathcal{D}^{\prime}$.

We shall prove that (1) can be reformulated, in operator terms, as

$$
(\mathrm{QSP})\left\{\begin{array}{l}
\frac{\mathrm{d} C u}{\mathrm{~d} t}(t)+A u(t)+\varepsilon^{2} B u(t)=f(t), \quad \text { in } V^{\prime} \\
C u(0)=C u_{0}
\end{array}\right.
$$

where the operators $A, B, C$ of $\mathcal{L}\left(V, V^{\prime}\right)$ are given by

$$
\begin{array}{ll}
\langle A u, v\rangle=a(u, v) & \forall u, v \in V, \\
\langle B u, v\rangle=b(u, v) & \forall u, v \in V, \\
\langle C u, v\rangle=c(u, v) & \forall u, v \in V .
\end{array}
$$

Which seems to be more extraordinary is that, in fact, the quasi-static problem is well posed even for $\epsilon=0$ and that the stabilization, as $t \rightarrow+\infty$, holds on the space $V_{A}$ (see [2]) defined as the completion of $V$ with a norm defined by $A$ (and so, such that $\mathbf{u}_{\infty} \notin \mathcal{D}^{\prime}$ if, for instance, $\mathbf{f}_{\infty} \in V^{\prime}-C^{\infty}$ ).

As a matter of fact, we shall not work directly with the displacements $\mathbf{u}$ since it can be shown that the singular perturbation problem can be reduced to a formulation on its trace on a part of the boundary $\Gamma$ (here assumed as a one-dimensional compact manifold without boundary). The bilinear forms $a, b, c$ and the energy space $V$ must be also adapted to this trace formulation. We shall recall this, in Section 3, following the ideas of [6]. 


\section{A special sensitive evolution problem}

In this Section, for the sake of simplicity in the exposition, we shall assume that $\Gamma=S^{1}=\mathbb{T}$, the unit circle. So that, we can assume that any function defined on $\mathbb{T}$ is $2 \pi$-periodic in $x$.

The sequence of functions $\mathrm{e}^{i k x}$ can be used to describe any function $v$ in $L^{2}(\mathbb{T})$ (and its Fourier series)

$$
v=\sum_{-\infty}^{+\infty} v_{k} w_{k}
$$

with

$$
w_{k}(x)=\frac{1}{\sqrt{2 \pi}} \mathrm{e}^{i k x}
$$

As, $w_{k}$ is an orthonormal basis in $L^{2}(\mathbb{T})$, we have

$$
v \in L^{2}(\mathbb{T}) \quad \text { iff } \quad\|v\|_{L^{2}}^{2}=\sum_{k=-\infty}^{+\infty}\left|v_{k}\right|^{2}<\infty .
$$

Differentiating with respect to $x$ amounts to multiply any $v_{k}$ by $i k$, so the following equivalence holds

$$
v \in H^{1}(\mathbb{T}) \quad \text { iff } \quad\|v\|_{H^{1}}^{2}=\sum_{k=-\infty}^{+\infty}\left|v_{k}\right|^{2}\left(1+k^{2}\right)<\infty,
$$

and by duality

$$
v \in H^{-1}(\mathbb{T}) \quad \text { iff } \quad\|v\|_{H^{-1}}^{2}=\sum_{k=-\infty}^{+\infty} \frac{\left|v_{k}\right|^{2}}{\left(1+k^{2}\right)}<\infty .
$$

For $m>1, m \in \mathbb{N}$ we can use (over the space $H^{m}(\mathbb{T})$ ) the norm (equivalent to the one obtained from the condition $u \in H^{m}(\mathbb{T})$ iff $\left.u_{x} \in H^{m-1}(\Gamma)\right)$

$$
\|v\|_{H^{m}}^{2}=\sum_{k=-\infty}^{+\infty}\left|v_{k}\right|^{2}\left(1+k^{2 m}\right)
$$

and so, by duality

$$
\|v\|_{H^{-m}}^{2}=\sum_{k=-\infty}^{+\infty} \frac{\left|v_{k}\right|^{2}}{\left(1+k^{2 m}\right)} .
$$

Moreover, by interpolation the above equivalence can be extended to any $m \in \mathbb{R}$.

We can define

$$
H^{\infty}(\mathbb{T})=\bigcap_{p \in \mathbb{N}} H^{p}(\mathbb{T})
$$

and we have that, for a general manifold $\mathbb{T}$

$$
\mathcal{D}(\mathbb{T}) \subset H^{\infty}(\mathbb{T}) \subset C^{\infty}(\mathbb{T})
$$

So, by duality

$$
H^{-\infty}(\mathbb{T}):=\left(H^{\infty}(\mathbb{T})\right)^{\prime} \subset \mathcal{D}^{\prime}(\mathbb{T})
$$

Notice that, in our case, since $\mathbb{T}$ is bounded, $\mathcal{D}(\mathbb{T})=H^{\infty}(\mathbb{T})=C^{\infty}(\mathbb{T})$.

We consider now a linear smoothing operator, $S: \mathcal{D}^{\prime}(\mathbb{T}) \rightarrow \mathcal{D}(\mathbb{T})$. For instance we can define

$$
S\left(\sum_{k=-\infty}^{+\infty} v_{k} w_{k}\right)=\sum_{k=-\infty}^{+\infty} v_{k} \mathrm{e}^{-\frac{|k|}{2}} w_{k} .
$$


Then we get that $\left.S\left(H^{s}(\mathbb{T})\right) \subset H^{r}(\mathbb{T})\right)$ for any $s, r \in \mathbb{R}^{+}$(in particular $S\left(\mathcal{D}^{\prime}(\mathbb{T})\right) \subset C^{\infty}(\mathbb{T})$ ).

More generally, we can take as $S$ any (linear) smoothing operator, i.e. $S\left(H^{s}(\mathbb{T})\right) \subset H^{r}(\mathbb{T})$, for any $s, r \in \mathbb{R}^{+}$. We assume that $S$ is injective

$$
v \in V, \quad S v=0 \Longrightarrow v=0
$$

We then define on $V$ the hermitian form

$$
a(u, v)=\int_{\mathbb{T}} S u \overline{S v} \mathrm{~d} x
$$

and the operator $A$, of $\mathcal{L}\left(V, V^{\prime}\right)$ given by

$$
\langle A u, v\rangle=a(u, v) \quad \forall u, v \in V .
$$

Notice that $A$ can be written in the form

$$
A=S^{*} S
$$

where we understand that $S$ and its adjoint $S^{*}$ are considered as

$$
S \in \mathcal{L}\left(H^{m}, H^{0}\right), \quad S^{*} \in \mathcal{L}\left(H^{0}, H^{-m}\right) .
$$

for some fixed (but arbitrary) $m \in \mathbb{R}^{+}$.

Lemma 1 The operator $A \in L\left(V, V^{\prime}\right)$ is injective.

Proof. Let $v \in V$ be such that $A v=0$. Then

$$
0=\langle A v, v\rangle=a(v, v)=\|S v\|_{0}^{2},
$$

and from the injectivity of $S$ we obtain $v=0$.

Now, we follow an idea already adopted in [2], which will play an important role also in the next Section. The previous lemma allows to define the following norm

$$
\|v\|_{V_{A}}=\|A v\|_{V^{\prime}}
$$

and we denote by $V_{A}$ to the completion of $V$ with $\|\cdot\|_{V_{A}}$. By Lemma 2.3 and Lemma 2.4 of [9] we have that the range of $A, \mathcal{R}(A)$, is dense in $V^{\prime}$ and that the operator $A$ extends as an isomorphism from $V_{A}$ onto $V^{\prime}$ (which we denote again as $A$ ). In particular, for any $F \in V^{\prime}$ there exists a unique solution $u \in V_{A}$ of $A u=F$.

We pass now to the consideration of the evolution problem

$$
\text { (EP) }\left\{\begin{array}{l}
\frac{\mathrm{d} u}{\mathrm{~d} t}(t)+A u=f_{\infty} \quad t>0 \\
u(0)=0
\end{array}\right.
$$

where

$$
f_{\infty} \in H^{0}
$$

i.e. we know that

$$
f_{\infty}=\sum_{-\infty}^{+\infty} b_{k} \mathrm{e}^{i k x} \quad \text { with } \sum_{k=-\infty}^{+\infty}\left|b_{k}\right|^{2}<\infty
$$


If we assume that $u(t, x)=\sum_{-\infty}^{+\infty} u_{k}(t) w_{k}$ satisfies (EP) then we get

$$
\left(\mathrm{EP}_{k}\right)\left\{\begin{array}{l}
\frac{\mathrm{d} u_{k}}{\mathrm{~d} t}(t)+\mathrm{e}^{-|k|} u_{k}(t)=b_{k} \quad t>0 \\
u_{k}(0)=0
\end{array}\right.
$$

and so,

$$
u_{k}(t)=b_{k} \mathrm{e}^{|k|}\left(1-\mathrm{e}^{-\mathrm{e}^{-|k|} t}\right)
$$

It is not difficult to show that the function

$$
u(t, x)=\sum_{-\infty}^{+\infty} b_{k} \mathrm{e}^{|k|}\left(1-\mathrm{e}^{-\mathrm{e}^{-|k|} t}\right) w_{k}(x)
$$

is such that $u \notin L^{\infty}\left(0,+\infty: H^{-m}(\mathbb{T})\right)$ (for some arbitrarily fixed $m$ ) since

$$
\|u(t, \cdot)\|_{H^{-m}}^{2}=\sum_{-\infty}^{+\infty} \frac{\left|b_{k}\right|^{2} \mathrm{e}^{2|k|}\left(1-\mathrm{e}^{-\mathrm{e}^{-|k|} t}\right)^{2}}{\left(1+k^{2 m}\right)}
$$

is unbounded as $t \rightarrow+\infty$. This implies that there is no reasonable hope to pass to the limit in $H^{-m}(\mathbb{T})$ as $t \rightarrow+\infty$. To follow a different approach, we introduce the space $V_{A}$ as the completion of $V$ with $\|v\|_{V_{A}}=\|A v\|_{V^{\prime}}$ for $A=S^{*} S$. Then, the norm of the space $V_{A}$ is given by

$$
\|v\|_{V_{A}}^{2}=\sum_{k=-\infty}^{+\infty} \mathrm{e}^{-2|k|}\left|v_{k}\right|^{2}
$$

so that

$$
\|u(t, \cdot)\|_{V_{A}}^{2}=\sum_{-\infty}^{+\infty}\left|b_{k}\right|^{2}\left(1-\mathrm{e}^{-\mathrm{e}^{-|k|} t}\right)^{2} \leq\left\|f_{\infty}\right\|_{H^{0}}^{2}
$$

Moreover

$$
\left|\frac{\partial u(t, x)}{\partial t}\right|=\sum_{-\infty}^{+\infty} b_{k} \mathrm{e}^{-\mathrm{e}^{-|k|} t} \Longrightarrow\left\|\frac{\partial u(t, \cdot)}{\partial t}\right\|_{H^{0}}^{2} \rightarrow 0 \quad \text { as } t \rightarrow \infty .
$$

Then (at least), $u(t, \cdot) \rightarrow u_{\infty}$, weakly in $V_{A}$, as $t \rightarrow+\infty$, for some $u_{\infty} \in V_{A}$. As a matter of fact, if we write the (unique) solution $u_{\infty}$ of the stationary limit problem

$$
\text { (SP) } \quad A u_{\infty}=f_{\infty}
$$

as

$$
u_{\infty}=\sum_{-\infty}^{+\infty} u_{\infty, k} w_{k}
$$

we get that

and we see that

$$
A u_{\infty}=f_{\infty} \quad \text { iff } \quad u_{\infty, k}=b_{k} \mathrm{e}^{|k|}
$$

as $t \rightarrow+\infty$. Since

$$
u_{k}(t)=b_{k} \mathrm{e}^{|k|}\left(1-\mathrm{e}^{-\mathrm{e}^{-|k|} t}\right) \rightarrow u_{\infty, k}
$$

$$
\|u(t, \cdot)\|_{V_{A}}^{2} \rightarrow\left\|u_{\infty}\right\|_{V_{A}}^{2}
$$

we get that $u(t, \cdot) \rightarrow u_{\infty}$, strongly in $V_{A}$, as $t \rightarrow+\infty$.

Remark 1 This shows that, in fact, even if (for simplicity) $f(t)=f_{\infty} \in H^{m}(\mathbb{T})-C^{\infty}(\mathbb{T})$, for some arbitrarily fixed $m$, the associate solution of $(\mathrm{SP}), u_{\infty}$, is not a distribution $\left(u_{\infty} \notin \mathcal{D}^{\prime}(\mathbb{T})\right)$. 


\section{On some quasi-static like-shell problems}

We consider now a problem related to the evolution formulation for a thin shell with a viscoelastic constitutive law. Then, according G. Duvaut, and J. L. Lions [4] (Section 6, Chapter 3) and J. Sanchez-Hubert and E. Sanchez Palencia [10] we arrive to a formulation of the type

$$
\frac{\partial^{2} \mathbf{U}}{\partial t^{2}}+c\left(\frac{\partial \mathbf{U}}{\partial t}, \mathbf{v}\right)+a(\mathbf{U}, \mathbf{v})+\varepsilon^{2} b(\mathbf{U}, \mathbf{v})=\langle\mathbf{f}, \mathbf{v}\rangle
$$

for suitable test functions in the energy space $V$ and suitable bilinear forms $a, b$, and $c$. The so called quasi-static problem corresponds to

$$
c\left(\frac{\partial \mathbf{u}}{\partial t}, \mathbf{v}\right)+a(\mathbf{u}, \mathbf{v})+\varepsilon^{2} b(\mathbf{u}, \mathbf{v})=\langle\mathbf{f}, \mathbf{v}\rangle
$$

and, as mentioned at the Introduction, it provides a reasonable good approximation for the asymptotic behavior of $\mathbf{U}$ when $t \rightarrow+\infty$.

Motivated by the arguments mentioned in Section 2 and the shell type formulation taken in [10] and [6], a "trace general formulation" can be obtained by starting with, $p(x, D)$ and $q(x, D)$, be two elliptic pseudo differential operators of order $m \in \mathbb{R}^{+}$with real symbols $p(x, \xi)$ and $q(x, \xi)$ continuous and coercive on $V=H^{m}(\Gamma)$, i.e. satisfying

$$
\begin{array}{ll}
c\|v\|_{m} \leq\|p(x, D) v\|_{0} \leq C\|v\|_{m} & \forall v \in V, \\
c\|v\|_{m} \leq\|q(x, D) v\|_{0} \leq C\|v\|_{m} & \forall v \in V,
\end{array}
$$

with $c, C>0$. Let $S$ be a (linear) smoothing operator, i.e. $\left.S\left(H^{s}(\Gamma)\right) \subset H^{r}(\Gamma)\right)$ for any $s, r \in \mathbb{R}^{+}$, where now $\Gamma$ is a given one-dimensional compact manifold without boundary. We assume that $S$ is injective

$$
v \in V \quad S v=0 \Longrightarrow v=0 .
$$

We then define on $V$ the Hermitian forms

$$
\begin{aligned}
a(u, v) & =\int_{\Gamma} S u \overline{S v} \mathrm{~d} x \\
b(u, v) & =\int_{\Gamma} p(x, D) u \overline{p(x, D) v} \mathrm{~d} x, \\
c(u, v) & =\int_{\Gamma} q(x, D) u \overline{q(x, D) v} \mathrm{~d} x .
\end{aligned}
$$

We notice that here we are neglecting the oscillation term arising when taking into account the membrane effects. We start with an abstract formulation: given $\varepsilon \geq 0, u_{0} \in V$ and $f \in H^{1}\left(0, T: V^{\prime}\right)$ for any $T>0$, find $u \in L^{2}(0, T: V)$ with $\frac{\mathrm{d} u}{\mathrm{~d} t} \in L^{2}\left(0, T: V^{\prime}\right)$ such that for any $v \in V$

$$
(\mathrm{QSP})\left\{\begin{array}{l}
\frac{\mathrm{d}}{\mathrm{d} t} c(u(t), v)+a(u(t), v)+\varepsilon^{2} b(u(t), v)=\langle f(t), v\rangle, \\
u(0)=u_{0}
\end{array}\right.
$$

where the bracket denotes the duality between $V^{\prime}$ and $V$ (it may depend on the small parameter $\varepsilon \geq 0$ ). In order to show that the problem is well posed we shall need to make some additional assumptions (see Showalter [12] for other alternatives). It is useful to reformulate (QSP) in terms of operators

$$
(\mathrm{QSP})\left\{\begin{array}{l}
\frac{\mathrm{d} C u}{\mathrm{~d} t}(t)+A u(t)+\varepsilon^{2} B u(t)=f(t), \quad \text { in } V^{\prime}, \\
C u(0)=C u_{0},
\end{array}\right.
$$


where the operators $A, B, C$ of $\mathcal{L}\left(V, V^{\prime}\right)$ are given by

$$
\begin{array}{ll}
\langle A u, v\rangle=a(u, v) & \forall u, v \in V, \\
\langle B u, v\rangle=b(u, v) & \forall u, v \in V, \\
\langle C u, v\rangle=c(u, v) & \forall u, v \in V .
\end{array}
$$

Notice that they can be written in the form

$$
A=S^{*} S, \quad B=p^{*} p \text { and } C=q^{*} q,
$$

where we understand that $S$ and its adjoint $S^{*}$ are considered as

$$
S \in \mathcal{L}\left(H^{m}, H^{0}\right), \quad S^{*} \in \mathcal{L}\left(H^{0}, H^{-m}\right) .
$$

As in the previous section, the operator $A \in L\left(V, V^{\prime}\right)$ is injective, the operator $A$ extends to an isomorphism from $V_{A}$ onto $V^{\prime}$ (which we denote again as $A$ ), and, in particular, for any $F \in V^{\prime}$ there exists a unique solution $u \in V_{A}$ of $A u=F$.

From the assumptions on $q(x, D)$ we deduce, from Lax-Milgram's Lemma, that operator $C$ admits an inverse $C^{-1} \in \mathcal{L}\left(V^{\prime}, V\right)$ and so, by introducing $w:=C u$ we can reformulate problem (QSP) as to find $w \in C\left([0,+\infty): V^{\prime}\right)$ solution of the Cauchy problem

$$
(\widetilde{\mathrm{QSP}})\left\{\begin{array}{l}
\frac{\mathrm{d} w}{\mathrm{~d} t}(t)+A C^{-1} w(t)+\varepsilon^{2} B C^{-1} w(t)=f(t), \quad \text { in } V^{\prime} \\
w(0)=w_{0}
\end{array}\right.
$$

with $w_{0}:=C u_{0}$.

Lemma 2 Assume that

$$
\begin{gathered}
q(x, D) \text { commutes with } S \text { and } p(x, D), \\
S u=\overline{S u}, \quad p(x, D) u=\overline{p(x, D) u} \quad \text { and } \quad q(x, D) u=\overline{q(x, D) u} \quad \text { for any } V .
\end{gathered}
$$

Then, for any $\epsilon \geq 0$ the operator $\mathcal{A} \in L\left(V^{\prime}, V^{\prime}\right)$ defined as $\mathcal{A} w=A C^{-1} w+\varepsilon^{2} B C^{-1} w$, for any $w \in V^{\prime}$, is a maximal monotone operator on $V^{\prime}$. Moreover, $\mathcal{A}$ is self-adjoint and $\mathcal{A}=\partial \varphi$, the subdifferential of the convex lower semi-continuous function

$$
\varphi(w)=\frac{1}{2}\left\|\mathcal{A}^{1 / 2} w\right\|^{2} \quad \text { for any } w \in V^{\prime}
$$

Proof. We first notice that $\mathcal{A}$ is (single valued) defined in the whole Hilbert space $V^{\prime}$. The monotonicity of $\mathcal{A}$ comes from the fact that, denoting by $((\cdot, \cdot))$ the scalar product in $V^{\prime}$ and if $w:=C u=q^{*} q u$,

$$
((\mathcal{A} w, w))=\left\langle S^{*} S u+\varepsilon^{2} p^{*} p u, q^{*} q u\right\rangle=(q S u, q S u)_{H^{0}}+\varepsilon^{2}(q p u, q p u)_{H^{0}} \geq 0 .
$$

Moreover $\mathcal{A}$ is a maximal monotone operator since, for any $F \in V^{\prime}$ and $\lambda>0$, there exists a (unique) $w \in V^{\prime}$ solution of

$$
\mathcal{A} w+\lambda w=F .
$$

Indeed, this is equivalent to solve the equation

$$
A u+\varepsilon^{2} B u+\lambda C u=F,
$$

which has a solution (even if $\epsilon=0$ ) via Lax-Milgram' Lemma. Finally, $\mathcal{A}$ is a selfadjoint operator since operators $A$ and $C$ verify

$$
\langle A u, v\rangle=a(u, v)=\int_{\Gamma} S u \overline{S v} \mathrm{~d} x=\langle A v, u\rangle
$$




$$
\langle C u, v\rangle=c(u, v)=\int_{\Gamma} q(x, D) u \overline{q(x, D) v} \mathrm{~d} x=\langle C v, u\rangle
$$

and so,

$$
\left(\left(A C^{-1} w, v\right)\right)=\left(\left(w,\left(C^{-1}\right) A v\right)\right) \quad \text { for any } v, w \in V^{\prime} .
$$

Analogously $\left(\left(B C^{-1} w, v\right)\right)=\left(\left(w,\left(C^{-1}\right) B v\right)\right)$ for any $v, w \in V^{\prime}$. Then, by Proposition 2.5 of Brezis [1] $\mathcal{A}=\partial \varphi$.

Remark 2 In concrete examples, the commutativity assumption (6) holds when the pseudo-differential operators $p(x, D)$ and $q(x, D)$ are $x$-independent. Nevertheless, other assumptions (different to (6)) implying the monotonicity of $\mathcal{A}$ are possible since we can have $\left\langle S^{*} S u+\varepsilon^{2} p^{*} p u, q^{*} q u\right\rangle \geq 0$ for some appropriate $q(x, D), S$ and $p(x, D)$, not necessarily commuting between them.

Concerning the stabilization we have:

Theorem 1 Assume the conditions (6) and (7) of the above Lemma. For $u_{0} \in V$ and, $\epsilon \geq 0$ let $u \in$ $C([0,+\infty): V)$ be the (unique) solution of

$$
(\mathrm{QSP})\left\{\begin{array}{l}
\frac{\mathrm{d} C u}{\mathrm{~d} t}(t)+A u(t)+\varepsilon^{2} B u(t)=f(t), \quad \text { in } V^{\prime}, \\
C u(0)=C u_{0} .
\end{array}\right.
$$

Then:

i) if $\epsilon>0$ and $f(t)-f_{\infty} \in L^{1}\left(0,+\infty: V^{\prime}\right)$ for some $f_{\infty} \in V^{\prime}$ then $\lim _{t \rightarrow \infty} u(t)=u_{\infty}$ in $V$ with $u_{\infty}$ the unique solution of

$$
A u_{\infty}+\varepsilon^{2} B u_{\infty}=f_{\infty}
$$

ii) if $\epsilon=0$ and $f(t) \equiv f_{\infty}$ for some $f_{\infty} \in V^{\prime}$ then $\left\|\frac{\mathrm{d} C u}{\mathrm{~d} t}(t)\right\|_{V^{\prime}}=O\left(\frac{1}{t}\right)$ and $\lim _{t \rightarrow \infty} u(t)=u_{\infty}$ in $V_{A}$ with $u_{\infty} \in V_{A}$ the unique solution of $A u_{\infty}=f_{\infty}$.

ProOF. Part i) is a consequence of the application of Théorème 3.11 of [1] since, from the coercivity assumptions (5) on $p(x, D)$ and $q(x, D)$, the set $\left\{w \in V^{\prime}:\left\|\mathcal{A}^{1 / 2} w\right\|^{2}+\|w\|^{2} \leq C\right\}$ is (strongly) compact in $V^{\prime}$. To show part ii) we use that, as $f(t) \equiv f_{\infty}$, and problem (QSP) can be written as

$$
(\widetilde{\mathrm{QSP}})\left\{\begin{array}{l}
\frac{\mathrm{d} w}{\mathrm{~d} t}(t)+\partial \varphi(w(t))=f_{\infty}, \quad \text { in } V^{\prime} \\
w(0)=w_{0}
\end{array}\right.
$$

then we get that $\left\|\frac{\mathrm{d} C u}{\mathrm{~d} t}(t)\right\|_{V^{\prime}}=O\left(\frac{1}{t}\right)$ (see Theoreme 3.10 of [1]). In contrast to the case $\epsilon>0$, the compactness of the set $\left\{w \in V^{\prime}:\|\varphi(w(t))\|^{2}+\|w\|^{2} \leq C\right\}$ fails and we can not apply the abstract result implying the convergence in $V$. Nevertheless some direct arguments lead to the conclusion. Indeed, let $u_{\infty} \in V_{A}$ be the unique solution of $A u_{\infty}=f_{\infty}$. Then, $\lim _{n \rightarrow \infty} A u\left(t_{n}\right)=A u_{\infty}$ in $V^{\prime}$, which, by construction, implies that $\lim _{n \rightarrow \infty} u\left(t_{n}\right)=u_{\infty}$ in $V_{A}$. Moreover, from the uniqueness of the solution of $A u_{\infty}=f_{\infty}$ we get that the limit takes place for any $t \rightarrow+\infty$.

Acknowledgement. Research partially supported by the project MTM2005-03463 of the DGISGPI (Spain). The research of J.I. Díaz was also partially supported by the project CCG07-UCM/ESP-2787 of the DGUIC of the CAM and the UCM. 


\section{References}

[1] BREZIS, H., (1972). Opérateurs maximaux monotones et semigroupes de contractions dans les espaces de Hilbert, North-Holland, Amsterdam.

[2] Callerie, D., (1996). Etude génerale d'un type de problèmes raides et de perturbation singulière, Comptes Rendus Mathématique, 323, 835-840.

[3] DíAZ, J. I. AND SÁNCHEZ-PALENCIA, E., (2007). On slender shells and related problems suggested by Torroja's structures, Asymptotic Analysis, 52, 259-297.

[4] Duvaut, G. And Lions, J. L., (1972). Les Inéquations en Mécanique et en Physique, París, Dunod.

[5] Gelfand, I. M. And Shilov, G., (1964). Generalized functions, Acad. Press, New York-London.

[6] Egorov, Y. V., Meunier, N. And SAncheZ-Palencia, E., (2007). Rigorus and heuristic treatment of certain sensitve singular perturbations, Journal de Mathématiques Pures et Appliquées, 88, 123-147.

[7] HöRmAnder, L., (1985). The analysis of linear partial differential operators I-III, Springer-Verlag, Berlin.

[8] Lions, J. L. And Magenes, E., (1972). Non-homogeneous boundary value problems and applications, Springer-Verlag, New York.

[9] Meunier, N., Sanchez-Hubert, J. And SAnchez Palencia, E., (2007). Various kinds of singular perturbations, Annales mathematiques Blaise Pascal, 14, 199-242.

[10] Sanchez-Hubert, J. And Sanchez Palencia, E., (1997). Coques élastiques minces. Propriétés asymptotiques, Masson, Paris.

[11] Schwartz, L., (1950). Théorie des Distributions, Hermann, Paris.

[12] Showalter, R. E., (1996). Monotone operator in Banach space and nonlinear equations, American Mathematical Society, Philadelphia.

\section{Jesús Ildefonso Díaz}

Departamento de Matemática Aplicada Universidad Complutense de Madrid Plaza de las Ciencias, 3 , 28040 Madrid.

diaz.racefyneinsde.es

and

Real Academia de Ciencias Exactas, Físicas y Naturales

Valverde 22,

28004 Madrid

\section{Evariste Sanchez Palencia}

Laboratoire de Modélisation en Mécanique, Université Pierre et Marie Curie

4, place Jussieu,

F - 75252 Paris Cedex 05

sanchez@lmm.jussieu.fr

and

Académie des sciences

23, quai de Conti - 75006

Paris,

France 